\title{
Von Willebrand Factor Multimeric Assay in Acquired von Willebrand Disease Diagnosis: A Report of Experience from North Estonia Medical Centre
}

\author{
Marika Pikta ${ }^{1,2, \odot ~ V a l d a s ~ B a n y s ~}{ }^{3} \quad$ Timea Szanto $^{4} \quad$ Lotta Joutsi-Korhonen ${ }^{5}$ \\ Ines Vaide ${ }^{6} \quad$ Mirja Varik $^{7} \quad$ Anna-Elina Lehtinen ${ }^{8} \quad$ Paul Giangrande $^{9} \quad$ Edward Laane $^{6}$
}

${ }^{1}$ Department of Health Technologies, Tallinn University of

Technology, Tallinn, Estonia

${ }^{2}$ Department of Laboratory Medicine, North Estonia Medical Centre, Tallinn, Estonia

${ }^{3}$ Department of Physiology, Biochemistry, Microbiology and Laboratory Medicine, Faculty of Medicine, Institute of Biomedical Sciences, Vilnius University, Vilnius, Lithuania

${ }^{4}$ Research Program Unit of Systems Oncology, Helsinki University Hospital, Unit of Coagulation Disorders, Helsinki, Finland

${ }^{5}$ Department of Clinical Chemistry, Unit of Coagulation Disorders, HUSLAB Laboratory Services, Helsinki University Hospital and University of Helsinki, Helsinki, Finland

${ }^{6}$ Hemato-Oncology Clinic, Institute of Clinical Medicine, Tartu University, Tartu, Estonia

${ }^{7}$ Hematology Department, North Estonia Medical Centre, Tallinn, Estonia

${ }^{8}$ Department of Hematology, Unit of Coagulation Disorders, Helsinki University Hospital Comprehensive Cancer Center and University of Helsinki, Helsinki, Finland

${ }^{9}$ Green Templeton College, University of Oxford, Oxford, United Kingdom

\begin{abstract}
Address for correspondence Marika Pikta, MD, Department of Laboratory Medicine, North Estonia Medical Centre, 19 Sütiste Str, Tallinn, 13419, Estonia (e-mail: marika.pikta@regionaalhaigla.ee).
\end{abstract}

\begin{abstract}
Keywords

- acquired von Willebrand syndrome

- von Willebrand factor

- von Willebrand factor multimers
\end{abstract}

Objectives Acquired von Willebrand syndrome (AVWS) is a rare and frequently underdiagnosed bleeding disorder with an unknown prevalence. The diagnosis of AVWS is made based on laboratory investigations and the presence of clinical symptoms. Evaluation and management of affected patients are complex due to the need for multiple laboratory assays.

Materials and Methods Here, we describe the clinical and laboratory data of seven patients with a diagnosis of AVWS. All patients met the criteria for AVWS based on laboratory findings, bleeding symptoms, and the absence of any previous history of a bleeding disorder. Results In all cases, the laboratory findings, lack of bleeding anamnesis, and family history suggested the presence of AVWS. Von Willebrand factor multimeric analysis showed decreased high-molecular weight (HMW) multimers in six cases. Patients with lower HMW multimers experienced more severe bleeding complications.

Conclusions The diagnosis of AVWS is complex and requires extensive laboratory evaluation. Interdisciplinary collaboration and complex laboratory evaluations are of paramount importance for the early recognition of AVWS and optimal AVWS diagnosis as well as successful clinical management.

Note: The study was performed according to the Declaration of Helsinki and was approved by the Tallinn Medical Research Ethics Committee.

published online June 28,2021
DOI https://doi.org/

$10.1055 / \mathrm{s}-0041-1730818$

ISSN 0974-2727 (c) 2021. The Indian Association of Laboratory Physicians.

This is an open access article published by Thieme under the terms of the Creative Commons Attribution-NonDerivative-NonCommercial-License, permitting copying and reproduction so long as the original work is given appropriate credit. Contents may not be used for commercial purposes, or adapted, remixed, transformed or built upon. (https://creativecommons.org/licenses/by-nc-nd/4.0/). Thieme Medical and Scientific Publishers Pvt. Ltd. A-12, 2nd Floor, Sector 2, Noida-201301 UP, India 


\section{Introduction}

Acquired von Willebrand syndrome (AVWS) is a rare and frequently underdiagnosed bleeding disorder, mainly due to the broad spectrum of possible clinical and laboratory features affiliated with this condition. ${ }^{1}$ The mechanisms behind von Willebrand factor (VWF) abnormalities depend upon the type of underlying disorder and may include increased clearance; enhanced shear stress and subsequent proteolysis; inhibition of VWF functions; adsorption to the platelet surface; or, rarely, decreased synthesis. ${ }^{1}$

The definition of AVWS was published by the VWF subcommittee in 2000. ${ }^{2}$ A diagnosis of AVWS can be made based on the following criteria: the existence of a lack of previous lifelong bleeding incidents and relevant family history, clinical picture, and laboratory investigation results, ${ }^{3}$ for example, VWF levels and factor VIII (FVIII) coagulant activity (FVIII:C) are sometimes decreased, a reduced VWF function/antigen ratio can indicate the existence of functional disorders, even if the absolute activity is within the normal limit, a loss or decrease in high-molecular weight (HMW) multimers may also be observable. The prevalence of AVWS remains unknown and ${ }^{4}$ the evaluation and management of affected patients may be complex due to the need for multiple laboratory assays, especially in those in whom the underlying disease (e.g., prosthetic heart valve or essential thrombocythemia [ET]) necessitates antithrombotic therapy. The initial laboratory tests used to assess AVWS include VWF level, VWF activity, and FVIII activity assays. Further tests include VWF multimer analysis, which is a sensitive tool able to detect the structural abnormalities of VWF even in the context of normal VWF activity levels. The frequency of the detection of inhibitors, that is, antibodies against VWF, is low in AVWS. Before 2016, it was not possible to confirm a suspicion of AVWS in Estonia because of a limitation of available laboratory VWF assays, while, since 2016, all VWF-related screening assays have been available to clinicians ${ }^{5}$ and, recently, a semiautomated VWF multimer assay has been incorporated into routine clinical practice at the North Estonia Medical Centre (NEMC). ${ }^{6,7}$

Here, we describe the clinical and laboratory data of seven patients diagnosed with AVWS at NEMC.

\section{Materials and Methods}

\section{Patients}

We included all consequent patients referred to and assessed at NEMC from the January 1, 2016, to December 31, 2017, who met the criteria for an AVWS diagnosis based on laboratory findings and bleeding symptoms together with the absence of any previous history of a bleeding disorder. ${ }^{3}$

The most common clinical symptoms were easy bruising, epistaxis, menorrhagia, and bleeding complications after tooth extraction. The mean age of the patients was 57.4 years (range: 22-80 years). The study group included five women and two men with various underlying diseases such as non-Hodgkin's lymphoma (NHL), monoclonal gammopathy of undetermined significance (MGUS), ET, polycythemia vera
(PV), secondary polycythemia due to cardiovascular diseases, obstructive sleep apnea syndrome, and autoimmune thyroiditis.

All cases were discussed at interdisciplinary meetings between laboratory and clinical staff. This retrospective study was performed as a collaboration between NEMC and Helsinki University Hospital, HUSLAB laboratory services, Coagulation Disorders Unit in partnership with The Twinning Program of the World Federation of Hemophilia (WFH). The study was performed according to the Declaration of Helsinki and was approved by the Tallinn Medical Research Ethics Committee.

\section{Blood Sampling}

During this study, peripheral venous blood specimens were collected into K2-EDTA tubes (BD Vacutainer; BD Diagnostics, Plymouth, UK) for a complete blood count, $3.2 \%$ sodium citrate tubes (BD Vacutainer; BD Diagnostics) for coagulation assays, and hirudin blood tubes (Roche Diagnostics, Switzerland) for platelet aggregation evaluation.

\section{Laboratory Investigations}

Based on the laboratory assays available in Estonia, the diagnostic algorithm for von Willebrand disease (VWD)/ syndrome was adopted in this study. ${ }^{8}$ Initial laboratory evaluations included complete blood count (Sysmex XE-5000; Roche Diagnostics); prothrombin time (PT) (Neoplastine $\mathrm{Cl}$ Plus; Diagnostica Stago, Asnières-sur-Seine, France); partial thromboplastin time (APTT) (PTT-A; Diagnostica Stago), VWF antigen (VWF:Ag) (Liatest-VWF:Ag; Diagnostica Stago); FVIII:C determined by a one-stage, clot-based assay (Diagnostica Stago, France); and VWF activity measured as VWF binding to the glycoprotein Ib (GPIb) receptor on the platelet surface (VWF:GPIbM) (Innovance VWF Ac kit; Siemens Healthcare Diagnostics, Marburg, Germany). All parameters were measured on the STA-R Evolution analyzer (Diagnostica Stago) using commercial kits.

Mixing studies were conducted to determine the etiology of prolonged APTT; the APTT test was repeated on a mixture of the patient's plasma with normal plasma immediately and after incubation for two hours at $37^{\circ} \mathrm{C}$. Depending on correction, FVIII, FIX, FXI, FXII, or lupus anticoagulant tests were performed.

Platelet aggregation was measured in whole blood by an impedance multiplate aggregometer (Roche Diagnostics) using the RISTOhigh test (final concentration of ristocetin: $0.77 \mathrm{mg} / \mathrm{mL}$ ) and RISTOlow test (final concentration of ristocetin: $0.2 \mathrm{mg} / \mathrm{mL}$ ). For both, the measurements were performed within 180 minutes after venipuncture.

The multimeric pattern of VWF was evaluated using the new Hydragel 5 von Willebrand multimers assay (Sebia, Lisses, France). ${ }^{6,9-11}$ The detailed protocol has previously been described..$^{12}$ In May 2019, the VWF multimer analysis with 5VWF was accredited in the NEMC laboratory according to the ISO15189:2012 standard. Both the visual evaluation of the gels and densitometric analysis were performed. VWF multimers were classified as low-molecular weight, intermediate-molecular weight, or HMW multimers with densitometry. 


\section{Case Series}

The main characteristics of the study participants are shown in - Table 1. All patients had other bleeding episodes and no family history for bleeding disorders. The International Society on Thrombosis and Hemostasis-Bleeding Assessment Tool was used to score the risk of bleeding (data not presented).
Case 1. A 67-year-old female patient with a diagnosis of NHL from 2012 onward was referred for consultation with a suspected bleeding disorder. Three bleeding episodes were noted during a period of 6 months before a definite AVWS diagnosis was made. First, a severe bleeding episode had occurred related to puncture of the right maxillary sinus; then, 3 months later, she was admitted to the emergency

Table 1 Demographic and laboratory characteristics of the study participants

\begin{tabular}{|c|c|c|c|c|c|c|c|c|}
\hline & $\begin{array}{l}\text { Reference } \\
\text { ranges }\end{array}$ & Case 1 & Case 2 & Case 3 & Case 4 & Case 5 & Case 6 & Case 7 \\
\hline Diagnosis & & $\begin{array}{l}\text { Non- } \\
\text { Hodgkin's } \\
\text { lymphoma }\end{array}$ & $\begin{array}{l}\text { ET, JAK2 } \\
\text { (V617F) }\end{array}$ & $\begin{array}{l}\text { ET, JAK2 } \\
\text { (V617F) }\end{array}$ & PV & MGUS & $\begin{array}{l}\text { Secondary } \\
\text { erythrocy- } \\
\text { tosis due } \\
\text { to cardi- } \\
\text { ovascular } \\
\text { diseases and } \\
\text { obstructive } \\
\text { sleep apnea } \\
\text { syndrome }\end{array}$ & $\begin{array}{l}\text { Autoimmune } \\
\text { thyroiditis }\end{array}$ \\
\hline $\begin{array}{l}\text { Clinical } \\
\text { symptoms }\end{array}$ & & $\begin{array}{l}\text { Epistaxis, } \\
\text { bleeding } \\
\text { compli- } \\
\text { cations } \\
\text { after tooth } \\
\text { extraction }\end{array}$ & Menorrhagia & $\begin{array}{l}\text { Bleeding } \\
\text { compli- } \\
\text { cations } \\
\text { after tooth } \\
\text { extraction }\end{array}$ & $\begin{array}{l}\text { Bleeding } \\
\text { compli- } \\
\text { cations } \\
\text { after tooth } \\
\text { extraction }\end{array}$ & Epistaxis & Epistaxis & $\begin{array}{l}\text { Spontaneous } \\
\text { hematoma }\end{array}$ \\
\hline Age, gender & & $67 \mathrm{~F}$ & $33 \mathrm{~F}$ & $61 \mathrm{~F}$ & $60 \mathrm{~F}$ & $78 \mathrm{M}$ & $80 \mathrm{M}$ & $22 \mathrm{~F}$ \\
\hline PT (sec) & $11.5-14.5$ & 13.3 & 13.2 & 13.0 & 12.6 & 13.0 & 12.9 & 12.6 \\
\hline APTT (sec) & $29-38$ & 44 & 48 & 41 & 48 & 46 & 34.4 & 33.6 \\
\hline $\begin{array}{l}\text { APTTmix } 1: 1 \\
\left(0^{`}, 120^{`}\right)\end{array}$ & & Correction & Correction & Correction & Correction & Correction & NA & NA \\
\hline VWF:Ag (\%) & $50-160$ & 25 & 61 & 83 & 102 & 29 & 269 & 35 \\
\hline VWFGPIbM (\%) & $\begin{array}{l}46-146 \text { ( } 0 \\
\text { group) } \\
61-179 \text { (non-0) }\end{array}$ & 14 & 34 & 29 & 62 & 11 & 174 & 41 \\
\hline $\begin{array}{l}\text { VWFGPIbM/Ag } \\
\text { ratio }\end{array}$ & $>0.7$ & 0.56 & 0.55 & 0.35 & 0.61 & 0.38 & 0.65 & 1.25 \\
\hline FVIII:C \% & $60-150$ & 42 & 37 & 48 & 118 & 21 & 253 & 65 \\
\hline RISTOhigh (U) & $98-180$ & 12 & ND & ND & 151 & 38 & ND & 112 \\
\hline $\begin{array}{l}\text { WBC count } \\
10^{9} / \mathrm{L}\end{array}$ & $4-10$ & 5.6 & 14.9 & 12.5 & 15.2 & 4.2 & 8.1 & 7.7 \\
\hline $\begin{array}{l}\text { RBC count } \\
10^{12} / \mathrm{L}\end{array}$ & $\begin{array}{l}\text { M 4.5-6.0; } \\
\text { N 4.0-5.5 }\end{array}$ & 4.6 & 5.4 & 8.5 & 5.7 & 5.0 & 6.2 & 4.1 \\
\hline Hematocrit (\%) & $\begin{array}{l}\text { M 40-52; } \\
\text { N 36-47 }\end{array}$ & 40 & 46 & 50 & 47 & 46 & 57 & 38 \\
\hline $\begin{array}{l}\text { Platelet count } \\
10^{9} / \mathrm{L}\end{array}$ & $150-400$ & 245 & 1391 & 1120 & 785 & 224 & 142 & 326 \\
\hline VWF multimers & $\begin{array}{l}\text { Persons } \\
\text { without VWD } \\
\text { (21): Normal } \\
\text { distribution }\end{array}$ & $\begin{array}{l}\text { Loss of } \\
\text { HMWM }\end{array}$ & $\begin{array}{l}\text { Decrease of } \\
\text { HMWM }\end{array}$ & $\begin{array}{l}\text { Loss of } \\
\text { HMWM }\end{array}$ & $\begin{array}{l}\text { Decrease of } \\
\text { HMWM }\end{array}$ & $\begin{array}{l}\text { Decrease } \\
\text { of HMWM }\end{array}$ & $\begin{array}{l}\text { Decrease of } \\
\text { HMWM }\end{array}$ & $\begin{array}{l}\text { Normal } \\
\text { distribution }\end{array}$ \\
\hline LMWM (\%) & $15.3(11-23)$ & 50.9 & 33.1 & 58.3 & 32.5 & 49.1 & 35.1 & 13.8 \\
\hline IMWM (\%) & $\begin{array}{l}30.2 \\
(23.1-35.8)\end{array}$ & 38.3 & 39.4 & 33.5 & 39.1 & 19.1 & 35.9 & 25.0 \\
\hline HMWM (\%) & $\begin{array}{l}54.8 \\
(45.1-65.9)\end{array}$ & 10.8 & 27.5 & 8.3 & 28.4 & 31.8 & 29.0 & 61.2 \\
\hline
\end{tabular}

Abbreviations: HMWM, high-molecular-weight multimers; IMWM, intermediate-molecular-weight multimers; LMWM, low-molecular-weight multimers; NA, nonapplicable; ND, not determined; VWF: Ag, von Willebrand factor antigen; VWF: GPIbM, VWF activity assays using recombinant gain-of-function mutant GPIb fragments allowing for the spontaneous binding of VWF to the mutant GPIb without ristocetin. 
department due to recurrent bleeding after tooth extraction requiring tamponade and bleeding from the right nasal cavity requiring electrocauterization. The patient was treated with tranexamic acid during all bleeding events and continues to be followed-up in the hematology clinic.

Case 2. A 33-year-old female patient with heavy menorrhagia and high platelet count was investigated. She had no antithrombotic treatment. A diagnosis of ET with a positive finding for a JAK2 (V617F) mutation was made. Menorrhagia was caused by secondary von Willebrand syndrome, and treatment with tranexamic acid was prescribed for use during menstrual bleeding.

Case 3. A 61-year-old female patient was investigated after experiencing bleeding after tooth extraction lasting 2 days. A high blood platelet count suggested the possibility of chronic myeloproliferative disease together with secondary von Willebrand syndrome. Further investigations confirmed JAK2 (V617F)-positive ET. Cessation of bleeding symptoms was achieved after platelet count normalization with hydroxyurea treatment.

Case 4. A 60-year-old female patient with PV from 2000 onward was referred for additional examination and consultation before planned tooth extraction. She experienced bleeding complications 2 year earlier after the tooth extraction. She was treated with hydroxyurea, blood exfusion, and low-dose aspirin. She was advised to stop aspirin 5 days before her next planned tooth extraction. Prophylactic treatment with $10 \mathrm{mg} / \mathrm{kg}$ of tranexamic acid given intravenously (IV) was prescribed three times daily on the procedure day and also one day before and after the procedure.

Case 5. A 78-year-old male patient was consulted because of recurrent epistaxis, with a need for cauterization throughout 2 previous years. His complete blood count was normal. Biochemical investigation showed a monoclonal peak $(3.1 \mathrm{~g} / \mathrm{L})$ in the $\gamma$-globulin region. Immunoglobulin G kappa monoclonal protein was confirmed by immunofixation. The kappa/lambda free light-chain ratio was 5.2 (reference range: 0.26-1.65), compatible with a diagnosis of MGUS. Tranexamic acid was prescribed in the case of a bleeding episode and the patient remains under close follow-up observation by the hematology clinic.

Case 6. An 81-year-old male patient with cardiovascular disease and obstructive sleep apnea syndrome was referred to a hematologist by his general practitioner due to frequent epistaxis (nosebleeds) occurring in the 2 previous years, with the need for nasal tamponade at the emergency department. The complete blood count revealed an increased red blood cell count $\left(6.10^{12} / \mathrm{L}\right)$, increased hemoglobin level (176 g/L), and increased hematocrit concentration (54.9\%), which raised the suspicion for PV. However, further studies on BCR/ABL p210 and JAK2 V617F mutations were normal, supporting the diagnosis of secondary erythrocytosis due to cardiovascular disease, which is one condition that can cause AVWS. The patient was counseled, and instructions were given for handling future bleeding episodes. Tranexamic acid was also prescribed to treat further bleeding episodes.

Case 7. A 22-year-old female patient was referred to the hematologist for bleeding evaluation. She reported the development of apparently spontaneous subcutaneous hematomas, unrelated to trauma or physical activity, during the last 3 years. Additional examination showed increased thyroid-stimulating hormone (TSH) and thyroid peroxidase (> $1000 \mathrm{U} / \mathrm{mL}$ ) levels, consistent with a diagnosis of autoimmune thyroiditis, and the patient was referred to the endocrinologist. Her hypothyroidism was treated and, 1 year later, normal TSH values were recorded together with normalization of coagulation test findings for VWF:Ag (69\%), VWF:GPIbM (86\%), fibrinogen (2.58 g/L), and $\mathrm{CRV}(<1 \mathrm{mg} / \mathrm{L})$.

\section{Results}

\section{Coagulation Workup for AVWD Diagnosis}

In this case series, coagulation studies showed normal PT and prolonged APTT (Cases 1-5). Mixing study revealed corrections for both immediate and incubated APTT tests, indicating a mild deficiency of FVIII in Cases 1, 2, 3, and 5. FIX, FXI, and FXII levels were normal. Follow-up assessments demonstrated severely decreased $(<35 \%)$ VWF activity in four of seven patients ( - Table $\mathbf{1}$ ), fulfilling the criteria for VWD diagnosis. Both decreased VWF:Ag and VWFGPIbM levels in Cases 1 and 5 and normal VWF:Ag levels with low VWF:GPIbM levels in Cases 2 and 3 were observed. In patient 6 , the levels of VWF:Ag, VWFGPIbM, and FVIII:C were increased, while a decreased VWF function/antigen ratio (VWFGPIbM/VWF:Ag) was recorded. High-dose ristocetin-induced platelet aggregation was decreased in two patients (cases 1 and 5), while low-dose ristocetin-induced platelet aggregation was normal. In Case 7, the levels of VWF:Ag and VWFGPIbM were both decreased with a normal VWF function/antigen ratio. Complete blood count and platelet aggregation studies were normal.

VWF multimeric analysis ( - Figs. 1 B-F) revealed decreased HMW multimers, supporting AVWS in all instances (Cases 1-6). In Case 6, during the visual investigation of gel, we did not detect any abnormalities in the VWF pattern, yet densitometric data provided additional information about the VWF multimeric structure. Multimeric analysis ( - Fig. $1 \mathbf{H}$ ) showed a normal distribution pattern, suggesting type 1 AVWS. We noted that patients with lower HMW multimers by densitometric evaluation presented with more severe bleeding complications.

\section{Discussion}

We herein describe the clinical and laboratory data of seven patients with AVWS. All cases were discussed in a multidisciplinary meeting involving both clinical and laboratory experts. In all cases, the laboratory findings and lack of previous lifelong bleeding episodes and family history suggested AVWS.

Earlier studies have documented that MGUS, ${ }^{13-15} \mathrm{NHL},{ }^{16}$ $\mathrm{ET},{ }^{17,18}$ and autoimmune hypothyroidism ${ }^{19}$ are associated with AVWS. The pathogenesis of AVWS is variable but may have an overlapping mechanism among patients with different underlying disorders. ${ }^{4,20,21}$ 

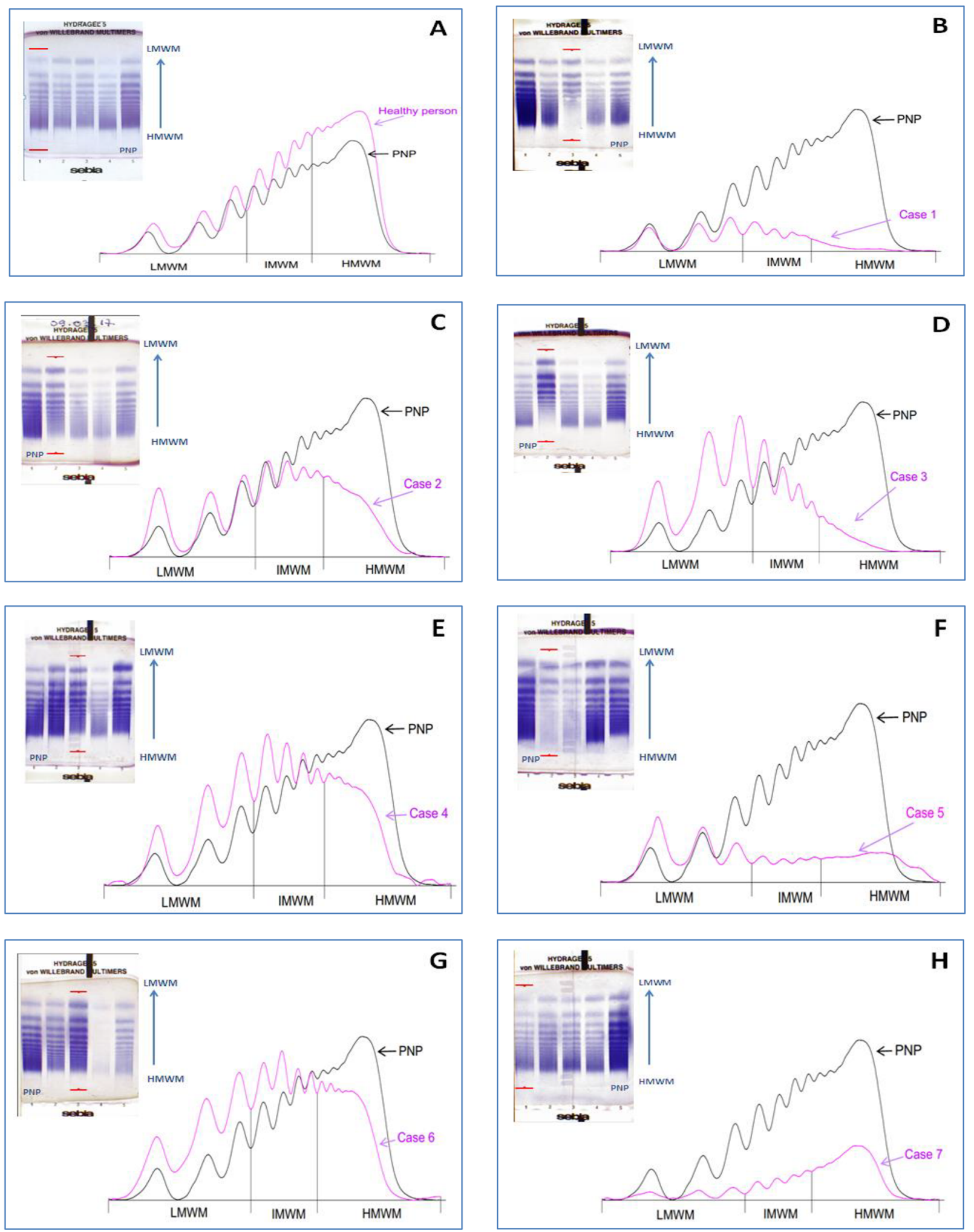

Fig. 1 Electrophoresis gels and densitograms: A-healthy person, B-Case 1, C-Case 2, D-Case 3, E-Case 4, F-Case 5, G-Case 6, H-Case 7. LMWM, low-molecular-weight multimers; IMWM, intermediate-molecular-weight multimers; HMWM, high-molecular-weight multimers; PNP, pool normal plasma.

In our series, six patients showed a type 2-like phenotype with decreased VWF activity to the Ag ratio and a loss/decrease of HMW multimers. One patient had a type
1 VWD phenotype. Recently developed diagnostic algorithms, based on standard laboratory assays, may assist clinicians in the diagnostic workup and help differentiate between AVWS 
and VWD types 1 and 2 . As reported by Federici et al, using data from the AVWS 2004 International Registry, ${ }^{22}$ AVWS is often correlated with a reduced ratio of VWF:RCo/VWF:Ag. The same findings were observed in our study in all cases except in Case 7, where the patient's clinical presentation was caused by the decreased synthesis of VWF.

Notably, our study demonstrated that VWF multimer analysis aids in the diagnosis of AVWS as an important, valuable tool. We further observed in our study that decreases in the level of VWFGPIbM and ratio of VWFGPIbM /VWF:Ag were associated with the selective loss or decrease of HMW multimers. Our study is in agreement with the report by Tiede et $\mathrm{al},{ }^{23}$ which suggested that a reduced VWF:RCo/Ag ratio in AVWS indicates inhibitory antibodies or a selective loss or decrease in HMW multimers. Separately, research conducted in Germany ${ }^{24,25}$ reported that, in 207 patients with cardiovascular disorders associated with AVWS and a loss of the HMW multimers, only $44 \%$ showed a ratio below 0.7 and noted that those patients would have likely been misdiagnosed without multimer analysis. In addition, VWF multimer analysis has been reported by Chen and Nichols as the most sensitive and specific method available for detecting such AVWS or acquired VWF abnormality without definite bleeding symptoms. ${ }^{26}$ In line with a previous study, ${ }^{27}$ our results revealed that AVWS is also associated with a decreased response to the higher ristocetin concentration (Cases 1 and 5).

The incidence of AVWS is possibly underestimated in the clinic. For example, as seen in the retrospective report by Mital et al on ET patients, AVWS may develop as frequently as in every fifth patient with ET. ${ }^{28}$ Furthermore, AVWS should be considered in all patients with new-onset bleeding whenever the laboratory findings suggest VWD, particularly in the presence of an AVWS-associated disorder. AVWS testing is also recommended prior to surgery or an intervention characterized by a high risk of bleeding in any individual with an AVWS-associated disorder. Treatment of the patient's underlying condition can lead to remission of AVWS. Strategies to prevent and/or treat bleeding episodes should also be put into place, including the use of VWF-containing FVIII concentrates, desmopressin, and tranexamic acid. Treatment success will depend largely upon the underlying pathogenesis of the disorder. Therefore, investigation of the VWF multimers presents profound clinical significance in suspected AVWS.

The gold standard for the detection of structural abnormalities of VWF is the multimeric assay. ${ }^{29}$ We assessed the VWF multimeric pattern in gels and quantified multimeric fractions using Sebia analysis software program. This method is easy to use and could prove very useful in future laboratory workup required for the diagnosis of AVWS. Interestingly, the densitometric evaluation of VWF multimers showed that patients with lower HMW multimer values presented with more severe bleeding complications. However, most AVWS patients do not bleed until they experience additional triggers like invasive procedures or trauma. ${ }^{4}$ Therefore, correct identification of patients with AVWS is a prerequisite for determining the applicable guidance on clinical management. ${ }^{30}$

\section{Conclusions}

Our data also demonstrate that the diagnosis of AVWS is complex and requires extensive laboratory evaluation. ${ }^{31}$ Our data support that VWF multimer analysis should be included in the AVWS diagnostic algorithm. Interdisciplinary collaboration and complex laboratory evaluations are of paramount importance for the early recognition of AVWS and the selection of appropriate clinical management protocols.

\section{Research Funding}

Sebia (Lisses, France) has donated the Hydragel 5 von Willebrand multimers kits.

\section{Conflicts of Interests}

None declared.

\section{Acknowledgments}

Research funding: Sebia (Lisses, France) donated the Hydragel 5 von Willebrand multimers kits. The authors would like to express gratitude to laboratory technicians Galina Trofimova and Tatjana Tverskaja for excellent technical assistance related to sample testing.

\section{References}

1 Michiels JJ, Budde U, van der Planken M, van Vliet $\mathrm{HH}$, Schroyens W, Berneman Z. Acquired von Willebrand syndromes: clinical features, aetiology, pathophysiology, classification and management. Best Pract Res Clin Haematol 2001;14(2):401-436

2 Federici $\mathrm{AB}$, Rand JH, Bucciarelli $\mathrm{P}$, et al. Subcommittee on von Willebrand Factor. Acquired von Willebrand syndrome: data from an international registry. Thromb Haemost 2000;84(2):345-349

3 James AH, Eikenboom J, Federici AB. State of the art: von Willebrand disease. Haemophilia 2016;22(Suppl 5):54-59

4 Collins P, Budde U, Rand JH, Federici AB, Kessler CM. Epidemiology and general guidelines of the management of acquired haemophilia and von Willebrand syndrome. Haemophilia 2008;14(Suppl 3):49-55

5 Pikta M, Zolotareva V, Tõnne J, Viigimaa M, Banys V. Implementation and verification of new VWF: Ac assay system with components from different manufacturers. Laboratorine Medicina 2016;72:185-188

6 Pikta M, Zemtsovskaja G, Bautista H, et al. Preclinical evaluation of a semi-automated and rapid commercial electrophoresis assay for von Willebrand factor multimers. J Clin Lab Anal 2018;32(6):e22416

7 Pikta M, Szanto T, Viigimaa M, et al. Evaluation of a new semi-automated hydragel 11 von Willebrand factor multimers assay kit for routine use. J Med Biochem 2021;40(2):167-172

8 Pikta M, Banys V, Vaide I, et al. Development of Diagnostic Algorithm for von Willebrand Disease within WFH the Twinning Tallinn-Helsinki program. Available at: https://www. postersessiononline.eu/173580348_eu/congresos/WFH2018/ aula/-M-P_139_WFH2018.pdf. Accessed December 9, 2020

9 Bowyer AE, Goodfellow KJ, Seidel H, et al. Evaluation of a semi-automated von Willebrand factor multimer assay, the Hydragel 5 von Willebrand multimer, by two European Centers. Res Pract Thromb Haemost 2018;2(4):790-799

10 Crist RA, Heikal NM, Rodgers GM, Grenache DG, Smock KJ. Evaluation of a new commercial method for von Willebrand factor multimeric analysis. Int J Lab Hematol 2018;40:586-591 
11 Favaloro EJ, Oliver S. Evaluation of a new commercial von Willebrand factor multimer assay. Haemophilia 2017;23(4):e373-e377

12 Oliver S, Lau KK, Chapman K, Favaloro EJ. Laboratory testing for von Willebrand factor multimers. Methods Mol Biol 2017;1646:495-511

13 Mannucci PM, Lombardi R, Bader R, et al. Studies of the pathophysiology of acquired von Willebrand's disease in seven patients with lymphoproliferative disorders or benign monoclonal gammopathies. Blood 1984;64(3):614-621

14 Federici AB, Stabile F, Castaman G, Canciani MT, Mannucci PM. Treatment of acquired von Willebrand syndrome in patients with monoclonal gammopathy of uncertain significance: comparison of three different therapeutic approaches. Blood 1998;92(8):2707-2711

15 Nitu-Whalley IC, Lee CA. Acquired von Willebrand syndrome-report of 10 cases and review of the literature. Haemophilia 1999;5(5):318-326

16 Tran-Thang C, Mannucci PM, Schneider P, Federici A, Bachmann F. Profound alterations of the multimeric structure of von Willebrand factor in a patient with malignant lymphoma. Br J Haematol 1985;61(2):307-314

17 Raman BKS, Sawdyk M, Saeed SM. Essential thrombocythemia with acquired von Willebrand's disease. Am J Clin Pathol 1987;88(1):102-106

18 Budde U, van Genderen PJ. Acquired von Willebrand disease in patients with high platelet counts. Semin Thromb Hemost 1997;23(5):425-431

19 Thorton JG, Parpia LA, Minford AMB. Hypothyroidism and von Willebrand's disease. Lancet 1987;1(8545):1314-1315

20 Mital A. Acquired von Willebrand Syndrome. Adv Clin Exp Med 2016;25(6):1337-1344

21 Franchini M, Lippi G. Acquired von Willebrand syndrome: an update. Am J Hematol 2007;82(5):368-375
22 Federici $A B$, Budde U, Rand JH. Acquired von Willebrand syndrome 2004: International Registry-diagnosis and management from online to bedside. Hamostaseologie 2004; 24(1):50-55

23 Tiede A, Priesack J, Werwitzke S, et al. Diagnostic workup of patients with acquired von Willebrand syndrome: a retrospective single-centre cohort study. J Thromb Haemost 2008;6(4):569-576

24 Budde U, Schneppenheim S, Dittmer R, Schneppenheim R. Classification of a large cohort of patients with von Willebrand disease between 2004 and 2010. Abstr. J Thromb Haemost 2011;9(suppl.2):56

25 Budde U. The multimer analysis. The clotting times. Spec Issue ECAT Found 2012;2:22-26

26 Chen D, Nichols WL. Von Willebrand disease testing repertoire: the past, present and future. The Clotting Times. Spec Issue ECAT Found 2012;2:16-21

27 Valarche V, Desconclois C, Boutekedjiret T, Dreyfus M, Proulle V. Multiplate whole blood impedance aggregometry: a new tool for von Willebrand disease. J Thromb Haemost 2011;9(8):1645-1647

28 Mital A, Prejzner W, Bieniaszewska M, Hellmann A. Prevalence of acquired von Willebrand syndrome during essential thrombocythemia: a retrospective analysis of 170 consecutive patients. Pol Arch Med Wewn 2015;125(12):914-920

29 Budde U, Pieconka A, Will K, Schneppenheim R. Laboratory testing for von Willebrand disease: contribution of multimer analysis to diagnosis and classification. Semin Thromb Hemost 2006;32(5):514-521

30 Tiede A, Rand JH, Budde U, Ganser A, Federici AB. How I treat the acquired von Willebrand syndrome. Blood 2011; 117(25):6777-6785

31 Shetty S, Kasatkar P, Ghosh K. Pathophysiology of acquired von Willebrand disease: a concise review. Eur J Haematol 2011; 87(2):99-106 